\title{
Mesh Movement for a Discrete-Adjoint Newton-Krylov Algorithm for Aerodynamic Optimization
}

\author{
Anh H. Truong,, Chad A. Oldfield,, and David W. Zingg \pm \\ University of Toronto, Toronto, Ontario M3H 5T6, Canada
}

DOI: $\underline{10.2514 / 1.33836}$

\begin{abstract}
A grid movement algorithm based on the linear elasticity method with multiple increments is presented. The method is relatively computationally expensive but is exceptionally robust, producing high-quality elements even for large shape changes. It is integrated with an aerodynamic shape optimization algorithm that uses an augmented adjoint approach for gradient calculation. The discrete-adjoint equations are augmented to explicitly include the sensitivities of the mesh movement, resulting in an increase in efficiency and numerical accuracy. This gradient computation method requires less computational time than a function evaluation and leads to significant computational savings as dimensionality is increased. The results of the application of these techniques to several large deformation and optimization cases are presented.
\end{abstract}

\begin{tabular}{lll} 
& \multicolumn{1}{c}{ Nomenclature } \\
$A$ & $=$ & coordinates of the airfoil surface \\
$E$ & $=$ & modulus of elasticity \\
$\mathbf{f}$ & $=$ & external forces \\
$G$ & $=$ & coordinates of the interior grid nodes \\
$\mathcal{J}, \mathcal{F}$ & $=$ & objective functions \\
$i$ & $=$ & increment number \\
$K$ & $=$ & stiffness matrix \\
$\mathcal{L}$ & $=$ & Lagrangian \\
$l$ & $=$ & length of a side of a triangle \\
$n$ & $=$ & number of increments \\
$P$ & $=$ & potential energy \\
$Q$ & $=$ & flow variables \\
$R$ & $=$ & radius of a circumscribed circle \\
$\mathcal{R}$ & $=$ & flow residual \\
$r$ & $=$ & residual of the grid movement equations \\
$s$ & $=$ & semiperimeter of a triangle \\
$\mathbf{u}$ & $=$ & element displacements \\
$V$ & $=$ & element volume \\
$X$ & $=$ & design variables \\
$\Gamma$ & $=$ & boundary \\
$\lambda, \psi$ & $=$ & adjoint vector \\
$\rho$ & $=$ & radius of an inscribed circle \\
$\sigma$ & $=$ & stress tensor \\
$\Phi$ & $=$ & element shape quality \\
$\Omega$ & $=$ & spatial domain \\
& &
\end{tabular}

Subscripts

$e \quad=\quad$ belonging to an element

$t=$ belonging to the entire system

$\left.\right|_{Q} \quad=\quad Q$ is held constant in the differentiation

$\Delta=$ subtriangular element inside a quadrilateral

Presented as Paper 3952 at the 18th AIAA Computational Fluid Dynamics Conference, Miami, FL, 25-28 June 2007; received 3 August 2007; revision received 8 February 2008; accepted for publication 11 February 2008. Copyright $@ 2008$ by David W. Zingg. Published by the American Institute of Aeronautics and Astronautics, Inc., with permission. Copies of this paper may be made for personal or internal use, on condition that the copier pay the $\$ 10.00$ per-copy fee to the Copyright Clearance Center, Inc., 222 Rosewood Drive, Danvers, MA 01923; include the code 0001-1452/08 \$10.00 in correspondence with the CCC.

${ }^{*}$ Graduate Student, Institute for Aerospace Studies, 4925 Dufferin Street. Student Member AIAA.

Graduate Student, Institute for Aerospace Studies, 4925 Dufferin Street.

Frofessor, Senior Canada Research Chair in Computational Aerodynamics, Institute for Aerospace Studies, 4925 Dufferin Street. Associate Fellow AIAA.

$$
\begin{array}{ll}
\text { Superscripts } \\
\hat{T} \quad=\quad \text { known variable on the boundary } \\
T \quad \text { transpose }
\end{array}
$$

\section{Introduction}

D YNAMIC meshes are required in the simulation of flow problems involving moving boundaries. These problems occur in many engineering applications, including blood flow in arteries; induced vibrations of suspension bridges, skyscrapers, and offshore structures; oscillating airfoils; aeroelastic response of wings; and unsteady motion of rotor blades in forward flight [1 $\underline{1} \underline{3}]$. More recently, with rapid advancements in processing speed and computing power and the extended use of computational fluid dynamics software as a design tool, dynamic meshes have also been used in the process of aerodynamic shape optimization. In either area of application, a new mesh has to be generated at each time step or iteration to fit the deformed surface, or the existing mesh has to be allowed to move with the computational domain. Allowing the existing mesh to evolve with the computational domain is generally more efficient than generating a new mesh. In shape optimization, the boundary surface undergoes many small changes; it would be too time consuming to regenerate the mesh in response to these deformations. Remeshing usually requires manual adjustments for complex geometries and the projection of the solution from the old mesh to the new one, because the new mesh may not have the same number of nodes and connectivity [4]. A mesh perturbation approach, on the other hand, will inherently preserve the original grid connectivity, hence ensuring the consistency of any mesh-induced errors in the flow solution (i.e., due to discretization error) between the initial and deformed grid, provided that a consistent mesh quality is maintained. It also ensures continuity in the sensitivity derivatives. The robustness and efficiency of the mesh deformation tool is particularly important in gradient-based optimization because any changes in the grid quality can have significant effects on these derivatives [5]. Most important, mesh movement algorithms have the potential to significantly reduce engineering cost by allowing the design process to be automated.

The focus of this work is on implementing a robust mesh movement method and efficient gradient computation for an aerodynamic optimizer developed by Nemec et al. [무요. The optimizer uses a Newton-Krylov algorithm for aerodynamic shape optimization on structured meshes with the discrete-adjoint method for the computation of the objective function gradient. The flow is governed by the two-dimensional compressible Navier-Stokes equations with the one-equation Spalart-Allmaras turbulence model and is solved using the preconditioned generalized minimal residual (GMRES) method in conjunction with an inexact Newton approach. 
The discrete-adjoint equation is solved using the same preconditioned GMRES method. The geometry is defined by a set of B-spline points, a subset of which is used as design variables. Geometric constraints are imposed on the objective function as penalty terms. It is shown that augmenting the discrete-adjoint equations to explicitly include the grid perturbation can result in significantly improved run time and gradient convergence.

\section{Background}

\section{Mesh Movement Methods}

A simple mesh movement technique for structured grids is to perturb each grid line running from the airfoil surface to the outer boundary individually. Burgreen et al. [9] computed the perturbation of each node on the grid line by linearly interpolating the perturbation between the airfoil and the far field. The method was later improved for highly curved grid lines by basing the interpolation on the arc length along the line [10]. In multiblock meshes, grid lines may not touch the airfoil or the far-field boundary, and the method needs to be modified. Nemec [7] uses trigonometric functions to preserve orthogonality for 2-D multielement airfoils. Jones and Samareh-Abolhassani [11] implemented an algebraic perturber that perturbs the block boundaries and interiors separately, using transfinite interpolation in an arc length parameter space. As they are strictly algebraic, these methods have a very short run time. However, for large perturbations, they can generate poor quality or tangled meshes. This can result in the need for the user to generate a new mesh during the optimization process. This is a notable deficiency, as an optimizer using such a method lacks complete automation.

A common technique is to model the mesh as a network of fictitious lineal springs whose stiffness is inversely proportional to the spring length. A linear system that represents this network of fictitious springs can be formulated and solved, yielding the interior nodal displacements due to a given boundary movement. Although it has been found to be fairly efficient and is applicable to unstructured or structured meshes, this approach can give tangled meshes for large shape changes. Farhat et al. [1] improved the robustness of the method by adding torsional springs. This was later extended to three dimensions [12]. Samareh [4] used quaternion algebra to improve the treatment of three-dimensional rotations and to help preserve the near-surface quality of two-dimensional viscous meshes. Recently, Liu et al. introduced a mesh deformation algorithm based on Delaunay graph mapping [13] that is comparable to the spring analogy method in robustness but surpasses it in efficiency, as it is noniterative and uses significantly less memory.

Another approach is to model the mesh as a continuum of elastic solid whose properties are defined by the modulus of elasticity and the Poisson ratio. Nodal movements are governed by the equations of linear elasticity, and mesh distortion can be controlled through the elements in the elastic matrix [14]. This method has been found to be much more robust than the spring analogy method, although relatively inefficient $[\underline{3}, \underline{5}, 15]$. Like the spring analogy technique, the use of the linear elasticity method usually requires some additional mesh stiffening mechanism to minimize the distortion of small elements, often by means of nonlinear material properties and/or nonlinear geometric deformation. Tezduyar et al. [16] introduced the technique of controlling the element deformation based on its size. This is achieved by assigning the modulus of elasticity to be inversely proportional to the cell volume. This way, smaller elements will be more rigid and more resistant to deformation. Stein et al. [14] and Bar-Yoseph et al. [3] augmented this method to include a stiffening mechanism that stiffens the mesh with increasing mesh displacement for cases with even larger amplitudes of displacement and rotation. The incremental approach is adopted here, wherein the deformed shape is achieved through a series of equal increments. At each increment, the stiffness is locally increased in highly strained areas. The result is a scheme that has a relatively high computational cost, yet is robust even to large shape changes.

\section{Gradient Evaluation Methods}

Although many optimization techniques make use of only the objective function value, the methods that are most computationally efficient are generally those that also use the value of the objective function gradient. Efficient gradient evaluation is essential to fast gradient-based optimizers.

The most straightforward way of computing a gradient is by means of finite differences. Although this has the advantage of simple implementation, it has limited accuracy due to truncation error for large step sizes and subtractive cancellation error for small step sizes. Additionally, the time required is long when compared with a flow solution, and the total time scales linearly with the number of design variables. The subtractive cancellation errors can be eliminated by using the complex step method. The theory for this method is laid out by Squire and Trapp [17], and its implementation is discussed by Martins et al. [18]. It allows very small step sizes to be used, thereby all but eliminating truncation error. However, the gradient calculation time is similar to that of finite differences.

Alternatively, the gradient can be computed analytically. Differentiated code can be produced quickly with algorithmic differentiation programs in either the forward or reverse modes. Griewank [19] provides a thorough discussion of the methods. In the forward mode, the differentiated code must be run once for each design variable, but in the reverse mode, the code must only be run once for each objective, and so it has a run time that is independent of the number of design variables. The speed advantages of the reverse mode are offset by a very large memory requirement: the intermediate values of each variable must be stored.

A semi-analytic approach to derivative calculation is the adjoint method. It was originally introduced to aerodynamic shape optimization by Pironneau [20] and Jameson [21]. As with reverse mode algorithmic differentiation, it provides a gradient computation time that is independent of the number of design variables; the time is typically on the order of the time taken by one function evaluation. The bulk of the computation involves solving a linear system whose size is independent of the number of design variables. The memory requirement is more reasonable than that of reverse mode algorithmic differentiation, but it takes considerable development time to hand code the derivatives required to form the adjoint equations.

The adjoint equations can be derived from the continuous or discretized flow equations. In the continuous approach, the adjoint equations are derived, discretized, then solved. Any discretization of the continuous adjoint equations may be used, and a carefully chosen discretization can result in computational savings. However, the computed gradients are different for each possible discretization of the adjoint system. This appears as an inconsistency between the computed gradient and the gradient one would expect when examining the discretized objective function; the discrepancy is on the order of the truncation error of the method and disappears as the meshes for the flow and adjoint solvers are refined.

For the discrete-adjoint approach, the process is reversed: the discrete-adjoint equations are derived from the discrete flow equations. The discrete-adjoint equations are one of the possible discretizations of the continuous ones and, by construction, this is the same discretization that was used for the objective function. The aforementioned error is therefore nil, and the gradient accuracy is regulated by the tolerance to which the equations are solved. As a result, the optimizer may be able to converge more tightly [22].

In accordance with a philosophy favoring tightly converged solutions while maintaining the speed advantages of the adjoint method, the discrete-adjoint method has been selected. For the optimization of the objective function $\mathcal{J}$ of the flow variables, $Q$, and the design variables, $X$, the objective function gradient can be evaluated as follows. First, the flow solver is converged, as represented by equating its residual to zero:

$$
\mathcal{R}(Q, X)=0
$$

Next, the adjoint vector, $\psi$, is found by solving the linear system 


$$
\left[\frac{\partial \mathcal{R}}{\partial Q}\right]^{T} \psi=-\left[\frac{\partial \mathcal{J}}{\partial Q}\right]^{T}
$$

where the derivatives are evaluated at the converged values of $Q$. Then, the gradient of the objective function is found by evaluating

$$
\frac{\partial \mathcal{J}}{\partial X}=\left.\frac{\partial \mathcal{J}}{\partial X}\right|_{Q}+\left.\psi^{T} \frac{\partial \mathcal{R}}{\partial X}\right|_{Q}
$$

where $\left.\right|_{Q}$ indicates that $Q$ is held constant in the differentiation. The solution of Eq. (2) represents the bulk of the computational work required in the gradient evaluation; it is the size of this system that is independent of the length of $X$, resulting in the speed benefits of the method.

\section{Mesh Sensitivities in the Discrete-Adjoint Method}

Considering that the evaluation of an aerodynamic objective function involves perturbing the mesh, it is to be expected that the sensitivities of the objective function to the design variables will involve sensitivities of the mesh perturbation algorithm. When evaluating the gradient using the discrete-adjoint method, Eqs. (2) and (3), these mesh sensitivities are implicitly included in the terms $\partial \mathcal{J} /\left.\bar{\partial} X\right|_{Q}$ and $\partial \mathcal{R} /\left.\partial X\right|_{Q}$

Within the discrete-adjoint method, a number of different approaches to computing grid sensitivities have been used. Kim et al. $[23,24]$ found that it is possible to neglect the grid sensitivities for design variables that do not involve the translation of a body and when the flow is considered to be inviscid.

Nemec and Zingg [6] and Martins et al. [25] used finite differences for partial derivatives with respect to the design variables, thus implicitly incorporating mesh sensitivities. Because their optimizers both use algebraic grid perturbation, it is not computationally onerous to repeatedly perturb the grid when forming the derivatives in this way. Burgreen and Baysal [10] and Le Moigne and Qin [26] hand differentiated arc length-based algebraic grid perturbers analytically. Bischof et al. [27] used automatic differentiation to reduce the human effort required to differentiate the more complicated algebraic grid perturbation method of Jones and Samareh [11].

More computationally expensive grid perturbation algorithms, such as the spring analogy and elasticity methods, have been differentiated using an adjoint approach. Maute et al. [28] presented an aeroelastic optimizer for three-dimensional Euler flows. They used the spring analogy mesh perturber of Farhat et al. [1]. The adjoint problem is posed as a large linear system that includes the coupled aerodynamic, structural, and mesh movement terms. It is solved using a staggered procedure, giving adjoint variables for each of the flow, grid perturbation, and structural solvers. The method is shown to be efficient, but it is noted that both the time and memory requirements for the derivative of the flow residual with respect to the interior node locations are considerable.

Recently, Nielsen and Park [29] presented an adjoint method for aerodynamic optimization. It computes an adjoint vector for the flow solver and then another for the grid perturber. This was performed for an unstructured grid using the linear elasticity mesh movement algorithm with one increment. The accuracy of the sensitivities was comparable to that obtained using direct differentiation, and the computational time was reduced dramatically. Mavriplis [30] took a similar approach using the spring analogy for grid perturbation. In this paper, we extend the method introduced by Nielsen and Park to allow multiple increments in the mesh movement algorithm.

Although mesh movement is more popular than regeneration in optimization, analytic and semi-analytic methods have also been used to differentiate grid generation codes. Sadrehaghighi et al. [31] used an algebraic grid generator to regenerate a grid at each optimizer iteration. Their sensitivity analysis includes analytic differentiation of the grid generation equations. Korivi et al. [32] provided grid sensitivities through algorithmic differentiation of the algebraic grid generator of Barger et al. [33]. For an aerodynamic sensitivity analysis, Pagaldipti and Chattopadhyay [34] differentiated the equations governing elliptic and hyperbolic grid generation, yielding a system of equations that can be solved for the sensitivities of the grid generator.

\section{Linear Elasticity Mesh Perturbation Algorithm}

\section{Solving for Displacement of Interior Nodes}

The displacement of the interior nodes in the spatial domain, $\Omega$, bounded by $\Gamma$, is governed by the equilibrium equation:

$$
\nabla \cdot \boldsymbol{\sigma}+\mathbf{f}=0 \quad \text { on } \Omega
$$

subject to the displacement boundary condition

$$
\mathbf{u}=\hat{\mathbf{u}} \quad \text { on } \Gamma
$$

where $\sigma$ is the stress tensor consisting of normal and shear stresses, $\mathbf{f}$ is the vector of external forces, $\mathbf{u}$ is the vector of element displacement, and $\hat{\mathbf{u}}$ is the vector of prescribed displacement on the boundary.

The constitutive equations connect the stress and strain field in the elastic body. For linear elasticity, the relation follows Hooke's law; the stress, $\boldsymbol{\sigma}$, is simply the product of the elasticity matrix, $\mathbf{C}$, and the strain vector, $\boldsymbol{\varepsilon}$ :

$$
\sigma=\mathbf{C} \varepsilon
$$

Assuming plane strain, isotropic materials, the elements in $\mathbf{C}$ can be expressed as a function of only two independent material constants, modulus of elasticity $E$ and Poisson's ratio $v$.

$$
\mathbf{C}=\frac{E}{(1+v)(1-2 v)}\left[\begin{array}{ccc}
1-v & v & 0 \\
v & 1-v & 0 \\
0 & 0 & 0.5-v
\end{array}\right]
$$

The strain, $\boldsymbol{\varepsilon}$, is related to the nodal displacement, $\mathbf{u}$, through the kinematic relation:

$$
\varepsilon=\mathbf{D u}
$$

The full matrix form of Eq. ( $\underline{8})$ is

$$
\boldsymbol{\varepsilon}=\left[\begin{array}{c}
\frac{\partial u}{\partial x} \\
\frac{\partial v}{\partial y} \\
\frac{\partial v}{\partial x}+\frac{\partial u}{\partial y}
\end{array}\right]=\left[\begin{array}{cc}
\frac{\partial}{\partial x} & 0 \\
0 & \frac{\partial}{\partial y} \\
\frac{\partial}{\partial y} & \frac{\partial}{\partial x}
\end{array}\right]\left[\begin{array}{c}
u \\
v
\end{array}\right]
$$

The modulus of elasticity is set to be proportional to the inverse of cell area at the $i$ th increment, $V^{(i)}$. In addition, a stiffening mechanism that varies with the distortion measures is applied to enhance control of the geometric distortion of the elements, including aspect ratio, taper, and skew. The change in stiffness from one increment to the next is based on cell distortion, defined as the ratio of the element shape quality, $\Phi$, to its reference value, such that $E$ increases to infinity as mesh entanglement is approached:

$$
E^{(i)}=\frac{1}{V^{(i)}}\left(\frac{\Phi_{e}^{(i)}}{\Phi_{e}^{(0)}}\right)^{2}
$$

For a quadrilateral element, the element distortion measure is defined in terms of the 2 norm of the element aspect ratios of its subtriangular elements:

$$
\Phi_{e}^{(i)}=\sqrt{\sum_{j=1}^{4}\left(\Phi_{\Delta_{j}}^{(i)}\right)^{2}}
$$

$\Delta_{j}$ is the subtriangular element inside the quadrilateral shown in Fig. 1. $\Delta_{1}=\Delta_{1,2,4}, \Delta_{2}=\Delta_{1,2,3}, \Delta_{3}=\Delta_{2,3,4}$, and $\Delta_{4}=\Delta_{1,3,4}$. The element aspect ratio of a triangular element is defined as

$$
\Phi_{\Delta}^{(i)}=\frac{R_{\Delta}}{\rho_{\Delta}}
$$




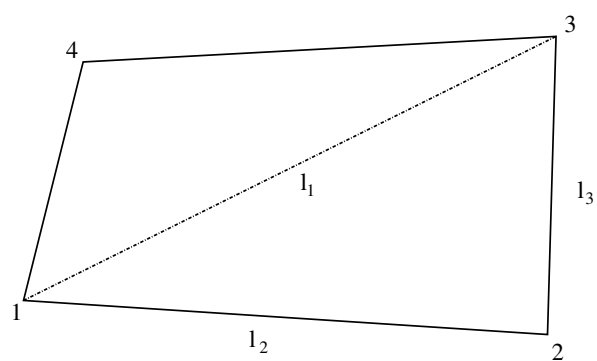

Fig. 1 Quadrilateral element with subtriangular element $\Delta_{123}$ and side lengths $l_{1}, l_{2}$, and $l_{3}$.

where $R_{\Delta}$ is the radius of the smallest circumscribed circle, and $\rho_{\Delta}$ is the radius of the largest inscribed circle:

$$
\frac{R_{\Delta}}{\rho_{\Delta}}=\frac{s_{\Delta} l_{1} l_{2} l_{3}}{4 A_{\Delta}^{2}}
$$

where $A_{\Delta}=\sqrt{s_{\Delta}\left(s_{\Delta}-l_{1}\right)\left(s_{\Delta}-l_{2}\right)\left(s_{\Delta}-l_{3}\right)}$ is the area of the triangle $\Delta, s_{\Delta}=0.5\left(l_{1}+l_{2}+l_{3}\right)$ is the semiperimeter, and $l_{1,2,3}$ are the lengths of the sides of the triangle. These formulations were suggested by Bar-Yoseph et al. [3] , and have been demonstrated to work well for large deformation problems.

The total strain energy stored in the elastic medium is given by

$$
P_{e}=\frac{1}{2} \sum_{e} \mathbf{u}_{e}^{T} K_{e} \mathbf{u}_{e}
$$

where $\mathbf{u}_{e}$ is an 8-element vector containing the $x$ - and $y$-direction displacements of each of the corner nodes of the quadrilateral element $e$ with stiffness $K_{e}$. This can also be written in terms of the total strain of the system:

$$
\frac{1}{2} \mathbf{u}_{t}^{T} K_{t} \mathbf{u}_{t}=P_{t}+\mathbf{u}_{t}^{T} \mathbf{f}_{t}
$$

The total strain energy is the sum of the potential energy, $P_{t}$, and the external work, $\mathbf{u}_{t}^{T} \mathbf{f}_{t}$, where $\mathbf{f}_{t}$ is the vector of external forces acting on each of the boundary nodes. $\mathbf{u}_{t}$ and $K_{t}$ are the displacement vector and stiffness matrix for the entire system. The force is known at the boundary, as it is the product of nodal displacement and stiffness. The dimension of these quantities is the number of degrees of freedom in the system ( $2 \times$ number of nodes in two dimensions). Each entry in $K_{t}$ is formed by summing the corresponding entries in each overlapping $K_{e}$, and $\mathbf{f}$ is zero except at the boundary where the displacement is known.

The steady solution of this system is such that the potential energy is minimized. This is obtained by setting the derivative of $P_{t}$ with respect to $\mathbf{u}_{t}$ to 0 , which yields the following linear system:

$$
K_{t} \mathbf{u}_{t}=\mathbf{f}_{t}
$$

For large deformations, the final deformation is achieved in a series of equal increments,

$$
A^{(i)}=A^{(0)}+\frac{i}{n}\left(A^{(n)}-A^{(0)}\right)
$$

where $A^{(i)}$ is the vector of coordinates on the airfoil surface for the $i$ th increment in a perturbation having a total of $n$ increments. $A^{(0)}$ is the parent airfoil, and $A^{(n)}=A$ is the fully perturbed airfoil.

For the purposes of the sensitivity analysis, the grid movement equations are written as setting a residual, $r^{(i)}$, to zero.

$$
0=r^{(i)}=r^{(i)}\left[G^{(i)}, G^{(i-1)}, A^{(i)}(X)\right]=K_{t}^{(i)} \mathbf{u}_{t}^{(i)}-\mathbf{f}_{t}^{(i)}
$$

where $G^{(i-1)}$ is the solution for the coordinates of the interior nodes at increment $i-1$.

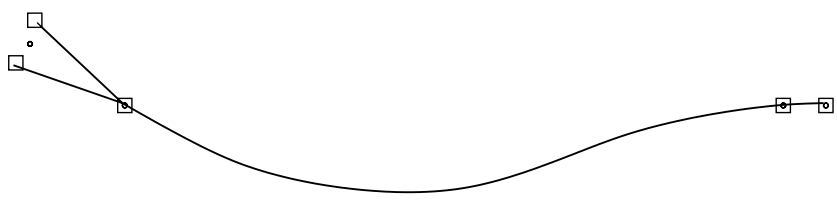

Fig. 2 Cubic polynomial fitting at the trailing edge.

deformation problems involving changes in the slope of the trailing edge, the location of this grid line is computed separately. The streamwise displacement of this grid line is determined using the algebraic method [7], whereas the normal displacement is obtained by fitting it to a cubic polynomial passing through four points: the midpoint between the two nodes on the upper and lower surface of the airfoil just before the trailing edge, the node at the trailing edge, and the last two nodes on the far-field boundary, as shown in Fig. 2. The special treatment of the trailing edge is used to prevent the rotational response of the small, elongated elements extending from the trailing edge as they resist deformation. The result is a smooth, gradual change in slope of the elements at the trailing edge propagating all the way to the far-field region.

\section{Augmented Adjoint Formulation}

Because the linear elasticity mesh movement method is much more expensive than the original algebraic mesh movement method, it is no longer practical or efficient to use finite differences to compute partial derivatives with respect to the design variables, which involves repeated mesh movement. The adjoint approach taken here augments the method of Nemec and Zingg through the explicit inclusion of mesh sensitivities in the adjoint equations. A rigorous method of deriving the discrete-adjoint equations is through the use of Lagrange multipliers, as presented by Gunzburger [35]. Using this method, the variables $X, G^{(i)}$, and $Q$ are considered to be independent. The optimization problem can then be posed as minimizing the design objective with respect to all of these variables, subject to the constraint that the flow solver and mesh movement algorithm must have converged. This is expressed as

$$
\begin{aligned}
& \min \mathcal{J}\left(Q, G^{(n)}, X\right) \quad \text { w.r.t. } Q, G^{(n)}, X \\
& \begin{array}{ll}
\text { s.t. } r^{(i)}\left[G^{(i)}, G^{(i-1)}, A^{(i)}(X)\right]=0, \quad i \in\{1,2, \ldots, n\} \\
\mathcal{R}\left(Q, G^{(n)}, X\right)=0
\end{array}
\end{aligned}
$$

To enforce the constraints, let the Lagrangian, $\mathcal{L}$, be defined as follows:

$$
\begin{aligned}
\mathcal{L} & =\mathcal{L}\left(\lambda^{(i)}, \psi, Q, G^{(i)}, X\right) \\
& =\mathcal{J}+\sum_{i=1}^{n} \lambda^{(i) T} r^{(i)}+\psi^{T} \mathcal{R} \quad i \in\{1,2, \ldots, n\}
\end{aligned}
$$

where $\psi$ and $\lambda^{(i)}$ are the Lagrange multipliers.

Setting each of the partial derivatives of the Lagrangian to zero then provides optimality conditions for the objective function:

$$
\begin{gathered}
\frac{\partial \mathcal{L}}{\partial \lambda^{(i)}}=0=r^{(i)}, \quad i \in\{1,2, \ldots, n\} \\
\frac{\partial \mathcal{L}}{\partial \psi}=0=\mathcal{R}
\end{gathered}
$$

$$
\frac{\partial \mathcal{L}}{\partial Q}=0=\frac{\partial \mathcal{J}}{\partial Q}+\psi^{T} \frac{\partial \mathcal{R}}{\partial Q}
$$

\section{Trailing-Edge Treatment}

For a C-grid topology, the grid line extending from the trailing edge to the far field is treated as a moving boundary. For large

$$
\frac{\partial \mathcal{L}}{\partial G^{(n)}}=0=\frac{\partial \mathcal{J}}{\partial G^{(n)}}+\lambda^{(n) T} \frac{\partial r^{(n)}}{\partial G^{(n)}}+\psi^{T} \frac{\partial \mathcal{R}}{\partial G^{(n)}}
$$




$$
\frac{\partial \mathcal{L}}{\partial G^{(i)}}=0=\lambda^{(i) T} \frac{\partial r^{(i)}}{\partial G^{(i)}}+\lambda^{(i+1) T} \frac{\partial r^{(i+1)}}{\partial G^{(i)}} i \in\{n-1, n-2, \ldots, 1\}
$$

$$
\frac{\partial \mathcal{L}}{\partial X}=0=\frac{\partial \mathcal{J}}{\partial X}+\sum_{i=1}^{n}\left(\lambda^{(i) T} \frac{\partial r^{(i)}}{\partial A^{(i)}} \frac{\partial A^{(i)}}{\partial X}\right)+\psi^{T} \frac{\partial \mathcal{R}}{\partial X}
$$

A number of approaches can be taken to find a solution to these optimality conditions to minimize the constrained objective, including solving for all of the variables simultaneously, an approach that defines simultaneous analysis and design. Using this one-shot approach, a large-scale solver sets the entire gradient of the Lagrangian to zero. This is not practical for multipoint optimization.

The sequential approach taken here delegates a great deal of the computation to the existing specialized solvers. For a given $X$, Eq. (20) can be solved using the mesh movement code to yield each $G^{(i)}$. Using that solution, the flow solver can be used to solve Eq. (21) to yield $Q$. The linear systems in Eqs. (22-24) can then be solved in the order they appear to give $\psi$ and each $\lambda^{(i)}$. Finally, the right-hand side of Eq. (25) can be evaluated to yield a value for $\partial \mathcal{L} / \partial X$.

This process sets most of the entries in the gradient of the Lagrangian to zero, leaving only $\partial \mathcal{L} / \partial X$ nonzero:

$$
\frac{\partial \mathcal{L}}{\partial\left\{\lambda^{(i)}, \psi, Q, G^{(i)}, X\right\}}=\left[\begin{array}{lllll}
0 & 0 & 0 & 0 & \frac{\partial \mathcal{L}}{\partial X}
\end{array}\right]
$$

The optimizer therefore only needs to consider $X, \mathcal{L}$, and $\partial \mathcal{L} / \partial X$. This can be denoted as the following objective function:

$$
\begin{aligned}
& \mathcal{F}(X)=\mathcal{L}, \quad\left\{\lambda^{(i)}, \psi, Q, G^{(i)} \mid \frac{\partial \mathcal{L}}{\partial\left\{\lambda^{(i)}, \psi, Q, G^{(i)}\right\}}=0\right\} \\
& i \in\{1,2, \ldots, n\}
\end{aligned}
$$

having a gradient given by

$$
\begin{gathered}
\frac{\partial \mathcal{F}}{\partial X}=\frac{\partial \mathcal{L}}{\partial X}=\frac{\partial \mathcal{J}}{\partial X}+\sum_{i=1}^{n}\left(\lambda^{(i) T} \frac{\partial r^{(i)}}{\partial A^{(i)}} \frac{\partial A^{(i)}}{\partial X}\right)+\psi^{T} \frac{\partial \mathcal{R}}{\partial X} \\
\left\{\lambda^{(i)}, \psi, Q, G^{(i)} \mid \frac{\partial \mathcal{L}}{\partial\left\{\lambda^{(i)}, \psi, Q, G^{(i)}\right\}}=0\right\}
\end{gathered}
$$

The function $\mathcal{F}$ and the gradient $\partial \mathcal{F} / \partial X$ are sent to the optimizer.
Notice that in evaluating the gradient, the adjoint equations, (22-24), are linear systems whose sizes are independent of the number of design variables. In evaluating the gradient, the only step whose computational time depends on the number of design variables is the evaluation of $\partial \mathcal{L} / \partial X$ in Eq. (25). This step requires only matrix multiplication and addition and so is not computationally demanding. The time required to evaluate the gradient should therefore be nearly independent of the number of design variables.

\section{Results}

Several cases involving pure mesh deformation and deformation in an optimization cycle are presented. The first two are pure large deflection cases consisting of translational and rotational deformations, demonstrating the robustness of the mesh movement algorithm. The next two are aerodynamic shape optimization cases examining the performance of the mesh movement algorithm with the augmented adjoint method compared with that of the original method. In the optimization cases, the wake cut is treated as part of the interior of the mesh rather than as a boundary defined by a cubic. The mesh movement problems are allowed to converge to machine zero using the conjugate gradient method with incomplete lowerupper preconditioning with zero fill. In these cases, the number of conjugate gradient iterations required varies from 20 to 900 , depending on the amount of deformation. The processing time per increment is typically $16 \%$ of the time required for a converged flow solution.

\section{Mesh Movement for Large Deformations}

Translational and rotational displacements are examined. In the first case, the RAE 2822 airfoil is deformed and given a translation of one chord length in both the $x$ and $y$ directions, as shown in Fig. 3 . The final configuration was achieved using only one increment, and the element quality [as defined in Eq. (11)] of the deformed mesh is equivalent to that of the original.

Figure $\underline{4}$ shows the result of rotating the NACA 0012 airfoil by $60 \mathrm{deg}$ about the trailing edge. The mesh has $201 \times 45$ nodes, and the final deformation is achieved using five increments. It can be observed that the smoothness and orthogonality of the original mesh are maintained even for such a large rotation, and the quality of smaller elements in the boundary layer of the airfoil is preserved. The deformation is propagated to larger elements in the far-field region. Again, using Eq. (11), the quality of the deformed mesh is determined to be $0.8 \overline{36}$, which is quite acceptable considering the large degree of rotation the mesh has undergone.

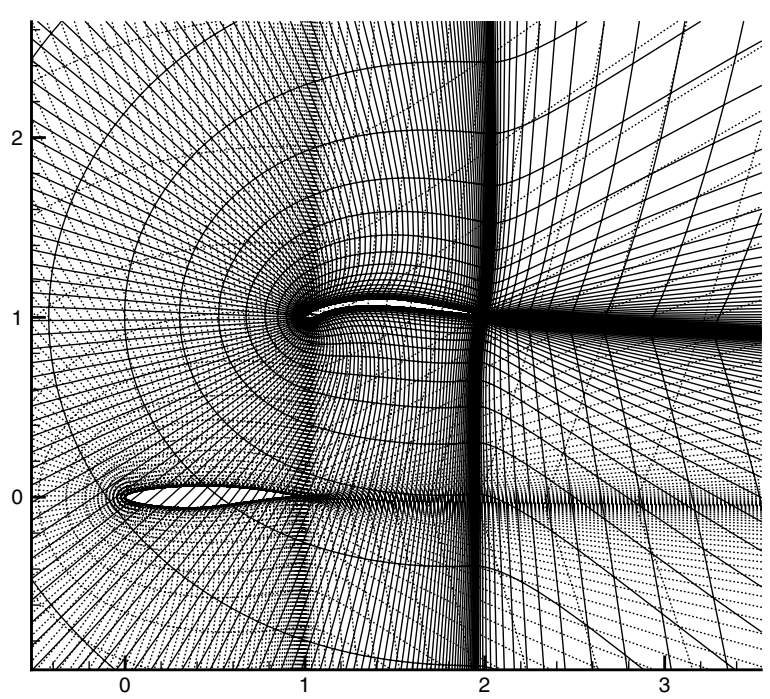

a) RAE 2822 airfoil $\mathrm{C}$ mesh

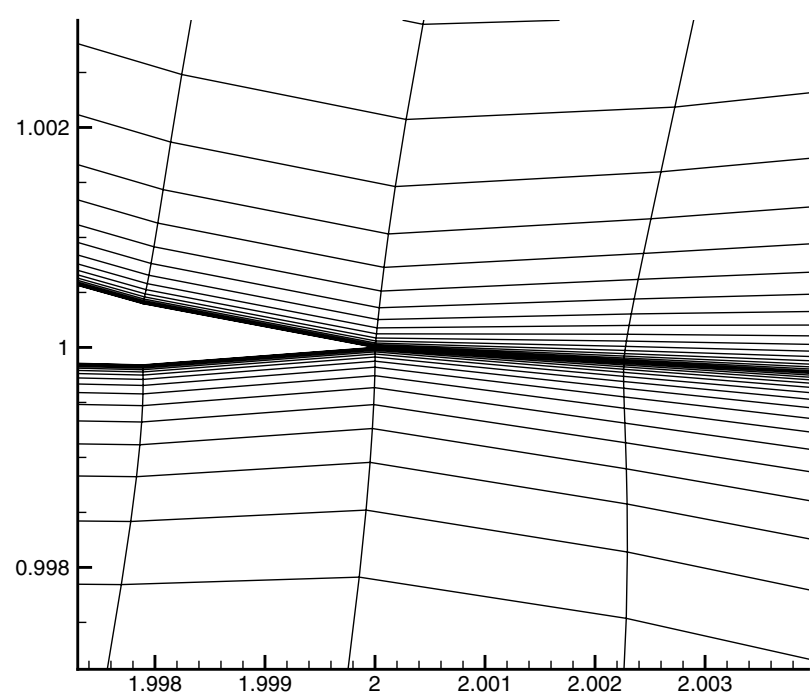

b) Modified mesh at the trailing edge

Fig. 3 Original and deformed mesh of the RAE 2822 airfoil consisting of 10,550 nodes. 


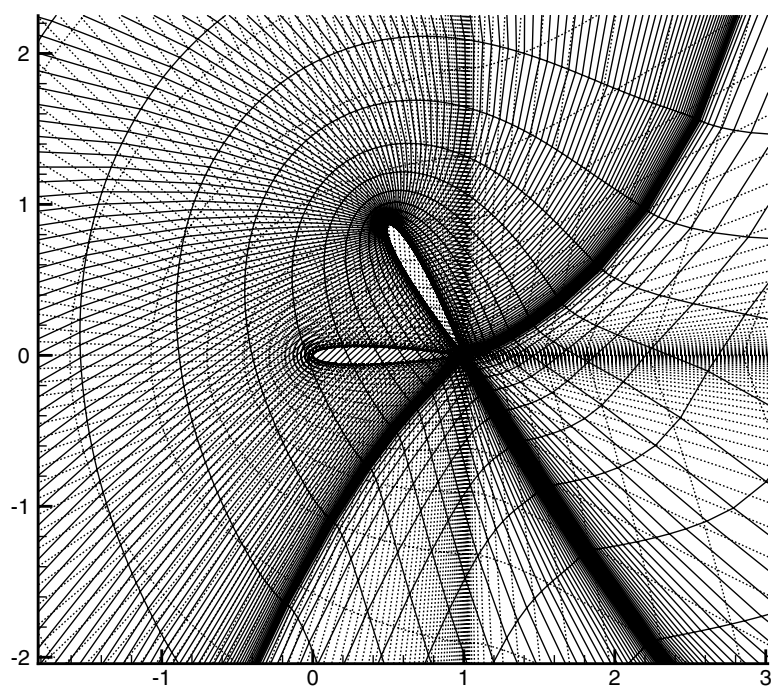

a) NACA 0012 airfoil $\mathrm{C}$ mesh

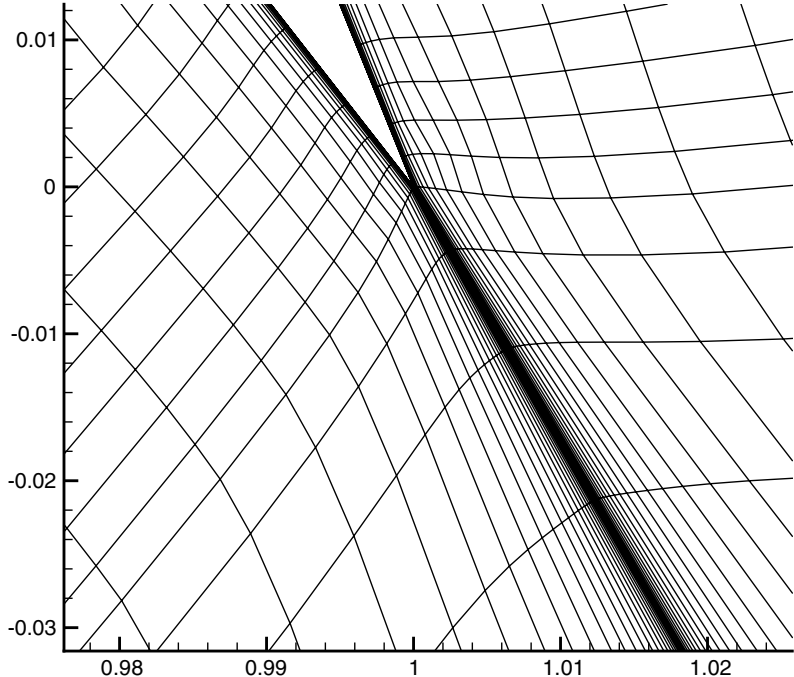

b) Modified mesh at the trailing edge

Fig. 4 Original and rotated mesh (by $60 \mathrm{deg}$ ) of the NACA 0012 airfoil consisting of 9,045 nodes.

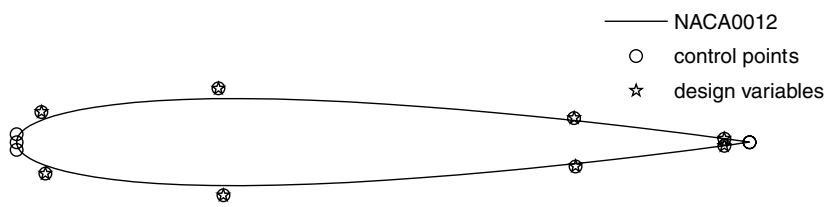

Fig. 5 Airfoil parameterization and design variables, cases A and B.

\section{Optimization Cases}

Test Case Details

Two test cases are presented. In case A, the design objective is to minimize the drag-to-lift ratio for an airfoil in a subsonic flow. The Mach number is 0.25 and the Reynolds number is $2.88 \times 10^{6}$. The turbulent flow is computed on a $225 \times 49$ mesh (11,025 nodes). The initial airfoil is the NACA 0012, which is parametrized by a B-spline curve having 13 control points, as shown in Fig. 5. The three control points at the leading edge and the two at the trailing edge are held fixed, whereas the $y$ coordinates of the remaining eight control points are used as design variables. The angle of attack, initially $4 \mathrm{deg}$, is also a design variable. Six minimum thickness constraints, given in Table 1, are used. A piecewise linear distribution for the radius of curvature constraint is given in Table 2 and is shown graphically in

Table 1 Thickness constraints (case A)

\begin{tabular}{lcc}
\hline \hline Chordwise coordinate & Thickness constraint & NACA 0012 thickness \\
\hline 0.05 & 0.04 & 0.0706 \\
0.35 & 0.11 & 0.1177 \\
0.65 & 0.04 & 0.0809 \\
0.75 & 0.03 & 0.0612 \\
0.85 & 0.026 & 0.0389 \\
0.95 & 0.012 & 0.0137 \\
0.99 & 0.002 & 0.0028 \\
\hline
\end{tabular}

Table 2 Radius of curvature constraint

\begin{tabular}{lcc}
\hline \hline $\begin{array}{l}\text { Chordwise } \\
\text { coordinate }\end{array}$ & $\begin{array}{c}\text { Radius of curvature } \\
\text { constraint }\end{array}$ & $\begin{array}{c}\text { NACA 0012 radius of } \\
\text { curvature }\end{array}$ \\
\hline 0 & 0.01 & 0.016 \\
0.005 & 0.01 & 0.030 \\
0.2 & 0.3 & 1.357 \\
0.8 & 0.3 & 7.528 \\
1 & 0.2 & 5.551 \\
\hline \hline
\end{tabular}

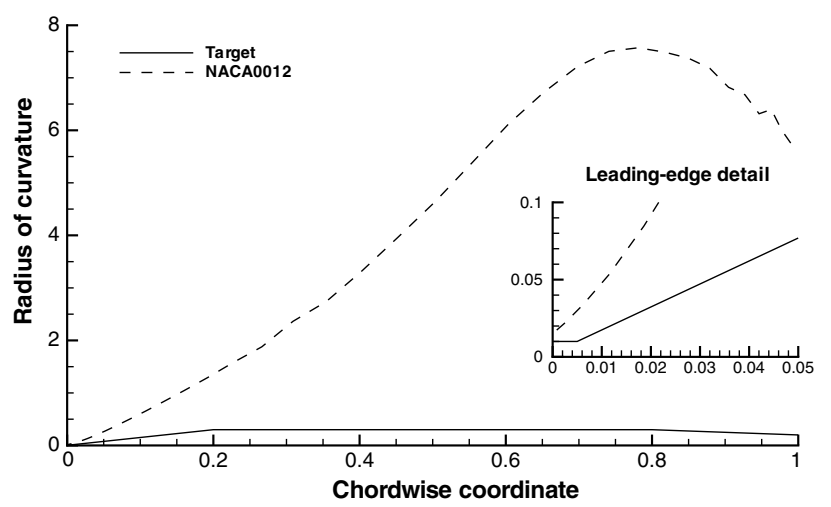

Fig. 6 Radius of curvature constraint.

Fig. 6. The respective properties of the NACA 0012 airfoil are included for reference. The minimum radius of curvature constraint was chosen to be less than the radius of curvature of the NACA 0012. In the critical areas of the nose and tail, the specified minimum radius of curvature is relatively close to the radius of curvature of the RAE 2822 airfoil.

In the second case, the design objective is to minimize drag at a fixed lift coefficient under transonic flow conditions. The Mach and Reynolds numbers are 0.78 and $2.0 \times 10^{7}$, respectively. The target lift coefficient is 0.75 . An enclosed minimum area constraint of 0.8 times the area of the initial airfoil, the NACA 0012, is enforced. Thickness constraints are 0.012 at a chordwise coordinate of 0.95 and 0.11 anywhere between chordwise coordinates 0.15 and 0.4 . The starting mesh as well as radius of curvature constraints are the same as in case A. The test cases were run using an Athlon64 3500+ processor with a clock speed of $2.2 \mathrm{GHz}$.

\section{Optimized Airfoil Performance}

For case A, the final lift-to-drag ratio achieved is 60.2, a substantial increase from the initial value of 31.1 , at an angle of attack of $4.7 \mathrm{deg}$. The optimized airfoils found using the augmented adjoint method, for case A, are shown in Fig. 7. Although the optimal airfoil shape does not depend much on the gradient calculation method, the figure shows that the shape is dependent on the grid perturbation technique used. The two variants of the elasticity method give very similar results, but the algebraic method gives a somewhat different shape.

To explore this, a new mesh was regenerated for each of the optimized airfoils, and the objective functions were recomputed on the new meshes. These objective functions include the constraint 


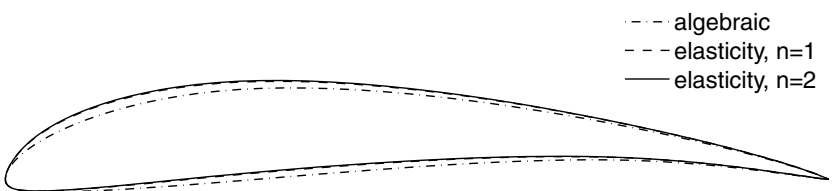

Fig. 7 Optimized airfoils for case A.

penalties; to maintain consistency, the penalties are not recomputed on the regenerated mesh, but are retained from the optimized result. These values, given in Table 3 , show that the elasticity method with $n=2$ provides the shape having the best performance, as computed on the regenerated mesh. Also, the comparison between the result computed on the perturbed and regenerated meshes is best for the elasticity method with $n=2$ and worst for the algebraic method. This suggests that the mesh obtained using the elasticity method with $n=2$ has the highest quality, that is, it is most similar to the original mesh.

For case B, the optimized airfoil (shown in Fig. 8) has a drag coefficient 14 counts lower than the original airfoil and is shock free, as can be seen in the pressure distribution shown in Fig. 9.

\section{Optimizer Convergence}

The convergence history for case A is shown in Fig. 10 for the algebraic mesh movement with and without the augmented adjoint and in Figs. 11 and 12 for the elasticity mesh movement with $n=1$ and 2 , respectively. The figures show the norm of the objective function gradient, and a normalization of the objective function, given by

$$
\mathcal{F}_{\text {normalized }}=\frac{\mathcal{F}}{\mathcal{F}_{\text {min }}}-1
$$

where $\mathcal{F}_{\text {min }}$ is the minimum objective function value found. This accentuates the difference between the objective function at a given iteration and the optimal value.

The curves for the normalized objective function show that the first two nonzero digits of the objective function value are determined after the first 15-20 iterations. At the end of the optimization, between 5 and 10 digits of the objective function are unchanging.

In all cases, the original and augmented adjoint methods are very similar for the first several iterations. As the optimization progresses, the differences between the methods accumulate and their convergence histories diverge. In the end, it can be seen that the gradient converges to about the same level for both gradient calculation methods, when using the algebraic grid perturbation scheme.

When the elasticity method is used to perturb the grid, however, the augmented adjoint method allows much tighter convergence of the gradient than does the original adjoint method (about 4 orders of magnitude difference). This is due to convergence difficulties experienced when using the original adjoint method and can be explained by gradient inaccuracy. Considering the gradient evaluation equation (3), in which the terms $\partial \mathcal{F} /\left.\partial X\right|_{Q}$ and $\partial \mathcal{R} /\left.\partial X\right|_{Q}$ are formed with finite differences, a great deal of error propagation can occur. Small amounts of numerical error are present in the calculation of $\mathcal{F}$ and $\mathcal{R}$ used in the finite differences; this is larger when the elasticity mesh perturbation method is used (rather

Table 3 Optimized airfoil performance using perturbed and regenerated meshes

\begin{tabular}{lccc}
\hline \hline & \multicolumn{3}{c}{ Objective function } \\
\cline { 2 - 4 } Perturbation method & Perturbed mesh & Regenerated mesh & Difference \\
\hline Algebraic & 0.016993 & 0.016685 & 0.000307 \\
Elasticity $(n=1)$ & 0.016694 & 0.016744 & 0.000050 \\
Elasticity $(n=2)$ & 0.016681 & 0.016668 & 0.000013 \\
\hline \hline
\end{tabular}



Fig. 8 Optimized airfoil for case B, linear elasticity $\operatorname{method}$ with $\boldsymbol{n}=\mathbf{2}$.

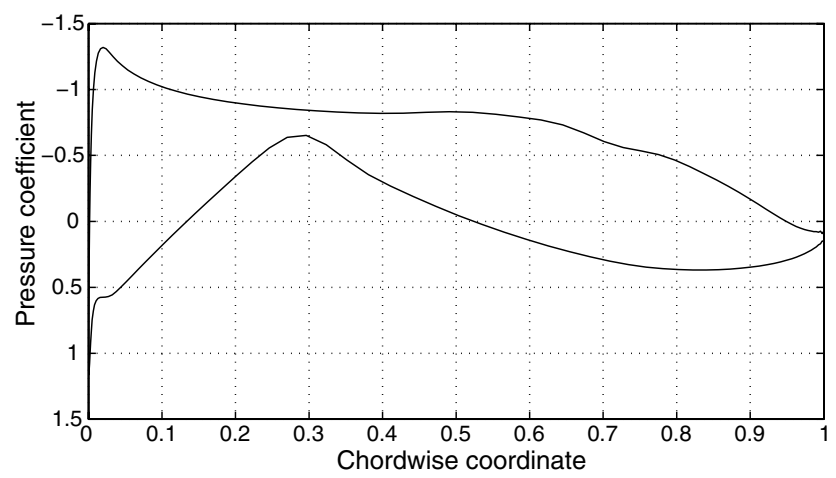

Fig. 9 Optimized pressure distribution for case $B$ at an angle of attack of $1.6 \mathrm{deg}, c_{l}=0.741$, and $c_{d}=0.013759$.

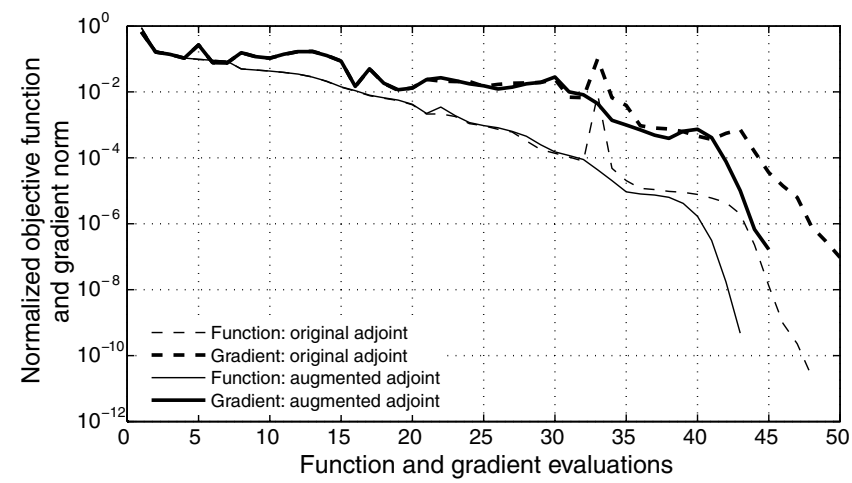

Fig. 10 Optimizer convergence for case A: algebraic method.

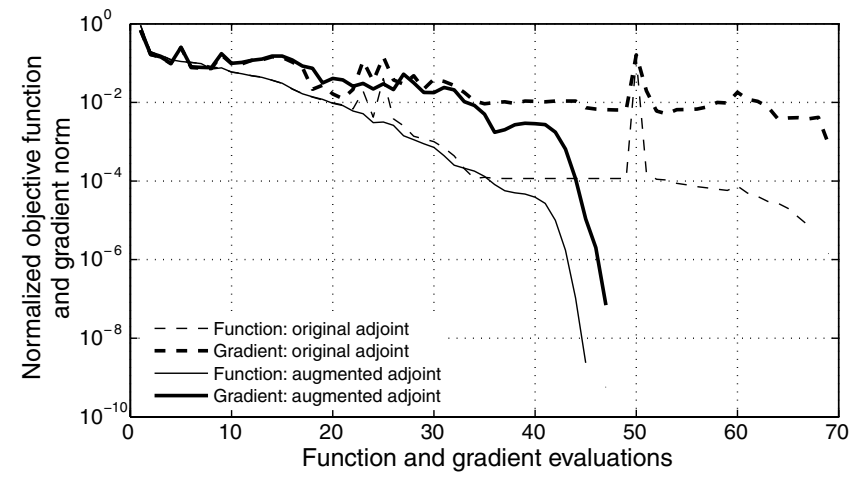

Fig. 11 Optimizer convergence for case A: elasticity method with $n=1$.

than the algebraic method), due to the imperfect convergence of the linear system. In the course of forming the finite differences, both truncation error and subtractive cancellation error occur. As a result, the derivatives have more erroneous trailing bits than do $\mathcal{F}$ and $\mathcal{R}$. The two derivatives are then added together. Near convergence of the optimizer, their sum (the gradient) is by definition a near-zero quantity. To effect this, several of the leading bits in $\partial \mathcal{F} /\left.\partial X\right|_{Q}$ and $\psi^{T} \partial \mathcal{R} /\left.\partial X\right|_{Q}$ must cancel, and so several more of the trailing bits become erroneous.

By this mechanism, small errors are amplified as the gradient is computed, and the amplification becomes more pronounced as optimizer convergence is reached. This eventually results in a 


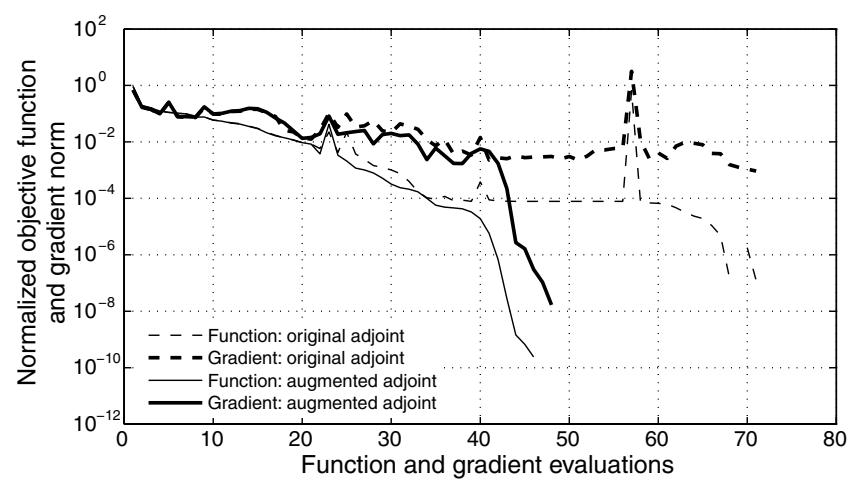

Fig. 12 Optimizer convergence for case A: elasticity method with $n=2$.

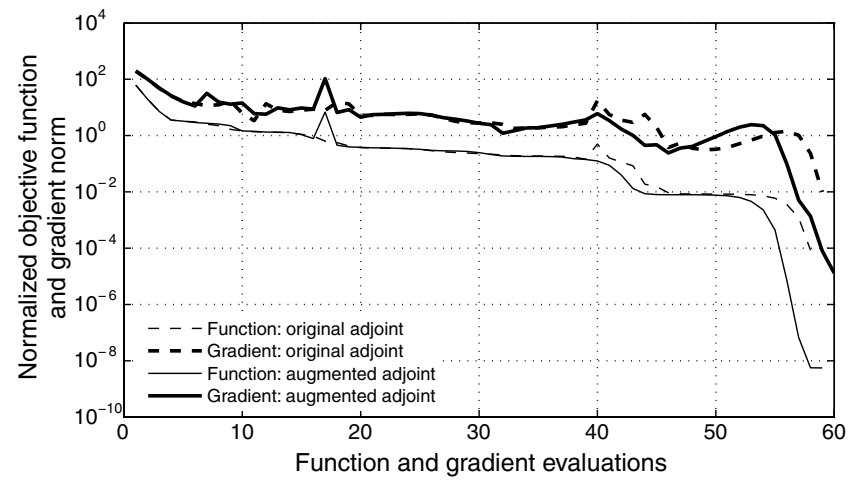

Fig. 13 Optimizer convergence for case B: elasticity method with $n=2$.

completely erroneous gradient, at which point the optimizer stalls. In the augmented adjoint method, however, finite differences are avoided. This elimination of one of the stages of error amplification results in a more accurate gradient, allowing tighter optimizer convergence.

Despite the fact that the convergence histories are different when using the original and augmented adjoint methods, they result in very similar optimized airfoils. The largest vertical displacement between the optimized airfoils is less than $10^{-6}$ chords when the algebraic grid perturbation is used. For the elasticity grid perturbation, the difference is less than $10^{-3}$ chords; this is expected, given the loose convergence obtained with the original adjoint method.

The optimizer convergence for case B shows trends similar to those found in case $\mathrm{A}$. The normalized function and gradient convergence for the case in which the elasticity method is used with $n=2$ is shown in Fig. 13. The figure shows that the augmented adjoint method converges about 3 orders of magnitude more tightly.

Using the same mesh movement method, the difference between the $y$ coordinates of the optimized airfoils obtained using the original and augmented adjoint methods is less than $10^{-4}$ chords (see Fig. 8). The difference between the airfoils obtained with different mesh movement methods is not significant in this case, less than $10^{-3}$ chords. This differs from case A, in which the algebraic mesh movement method gave a noticeably different airfoil. The reason for the difference is likely that shock elimination dominates the transonic problem, whereas viscous drag is more important for the subsonic airfoil and, thus, so is mesh orthogonality.

\section{Gradient Evaluation Time}

The CPU time required to evaluate the gradient was investigated, considering different mesh movement techniques, gradient evaluation methods, and numbers of design variables. In each of Figs. 14-16, the gradient evaluation time for the second iteration in case $\overline{\mathrm{A}}$ is plotted against the number of B-spline design variables, and the augmented adjoint method is contrasted with the original adjoint method. In Fig. 14, algebraic mesh movement is used. This shows

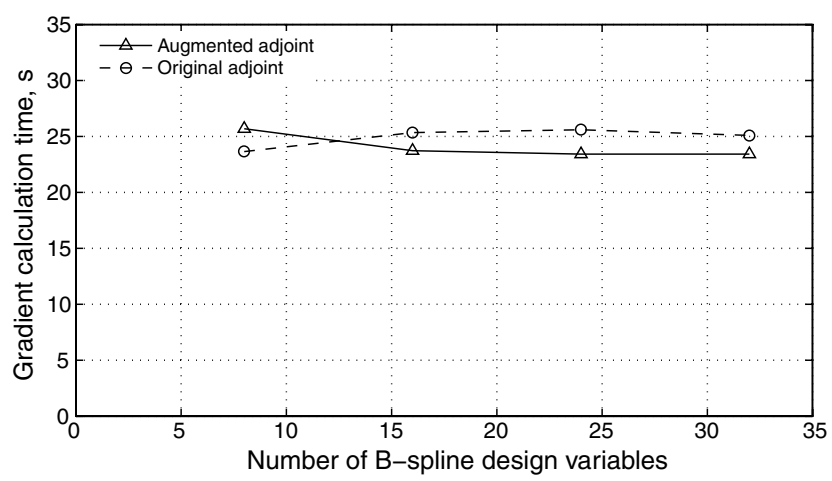

Fig. 14 Gradient calculation time for case A: algebraic method.

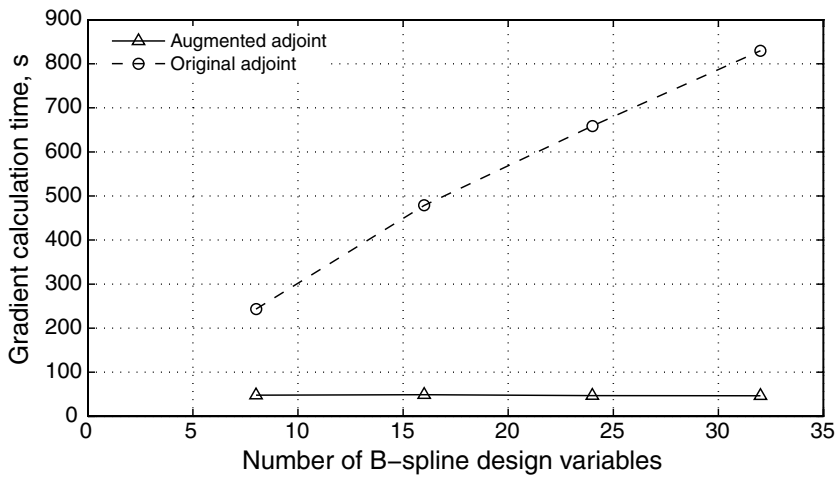

Fig. 15 Gradient calculation time for case A: elasticity method with $n=1$.

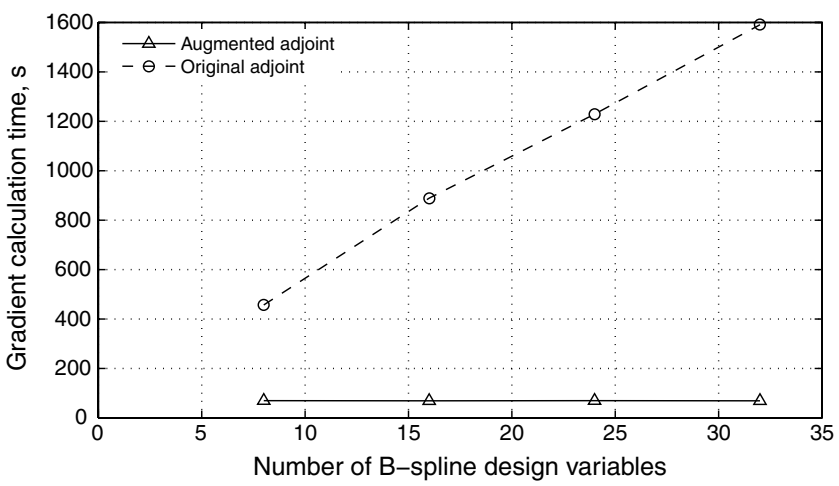

Fig. 16 Gradient calculation time for case A: elasticity method with $n=2$.

little difference between the two gradient evaluation techniques, although the original method is slightly slower. This difference is indicative of the speed of the analytic calculation of $\partial R / \partial G$. The time taken by the augmented method actually decreases some with an increasing number of design variables; this is likely noise due to the slightly different convergence of the solvers.

Figures 15 and 16 use the elasticity mesh movement method, with the number of increments, $n$, being 1 and 2, respectively. The time taken by the augmented method varies by less than $5 \%$ with differing numbers of design variables and does not show an increasing trend. Additionally, the augmented adjoint method is several times faster than the original adjoint method, whose time requirements increase linearly with the number of design variables. The evaluation time for the original adjoint method is several times longer than that of a flow solution, which takes roughly $50 \mathrm{~s}$.

In an entire optimization, the gradient evaluation time is essentially constant, despite warm starting each $\lambda^{(i)}$. The function evaluation (flow solution plus mesh movement) grows increasingly 
faster as the optimization progresses. This is due to warm starting; the flow solution gradually becomes faster, whereas the mesh movement accelerates abruptly near the end of the optimization. For the mesh densities used here, the flow solution requires about $50 \mathrm{~s}$ during the early iterations. The linear elasticity mesh movement requires about $8 \mathrm{~s}$ or $16 \%$ of the processing time required for the flow solution, per increment. Solving the flow adjoint equation requires $12 \%$ of the cost of the flow solution, whereas the solution of the grid adjoint equation requires $16 \%$ for each increment. If a function evaluation requires roughly $58 \mathrm{~s}$ for one increment, or $66 \mathrm{~s}$ for two, then the gradient calculation (flow adjoint equation solution plus mesh adjoint solution per increment) typically requires about $24 \%$ of the cost of a function evaluation for one increment and $33 \%$ for two increments.

\section{Conclusions}

We have presented a mesh movement technique that models the mesh through the equations of linear elasticity. To maintain mesh quality, cells are stiffened in inverse proportion to their area and quality. Multiple mesh movement increments can be used so that the stiffening can be adjusted based on intermediate quality measures. This mesh movement approach requires more computation than simpler techniques, such as algebraic methods, but is very robust, producing high-quality meshes even for large shape changes. An augmented adjoint approach is used to calculate adjoint variables associated with the mesh movement algorithm. Adjoint variables are computed for each increment used. The use of the augmented adjoint method allows the optimizer to converge much more tightly than was possible using the original adjoint method. This is indicative of increased gradient accuracy. The augmented adjoint method has a run time that is independent of the number of design variables. With the augmented adjoint approach, the cost of computing the gradient is about $24-33 \%$ of that of a function evaluation (mesh movement plus flow solution), depending on the number of increments.

\section{Acknowledgments}

This work was sponsored by the Natural Sciences and Engineering Research Council of Canada, the Canada Research Chairs Program, Bombardier Aerospace, the Society of Naval Architects and Marine Engineers, and the University of Toronto. Their financial support is gratefully acknowledged.

\section{References}

[1] Farhat, C., Degand, C., Koobus, B., and Lesoinne, M., "Torsional Springs for Two-Dimensional Dynamic Unstructured Fluid Meshes," Computer Methods in Applied Mechanics and Engineering, Vol. 163, Nos. 1-4, Sept. 1998, pp. 231-245. doi:10.1016/S0045-7825(98)00016-4

[2] Allen, C. B., "An Unsteady Flow Solver with Algebraic Grid Motion for Aeroelastic Simulations," ICAS 2002-R3, International Council of the Aeronautical Sciences, Bristol, England, U. K., 2002.

[3] Bar-Yoseph, P. Z., Mereu, S., Chippada, S., and Kalro, V. K., "Automatic Monitoring of Element Shape Quality in 2-D and 3-D Computational Mesh Dynamics," Computational Mechanics, Vol. 27, No. 5, May 2001, pp. 378-395. doi: $10.1007 / \mathrm{s} 004660100250$

[4] Samareh, J. A., "Application of Quaternions for Mesh Deformation," NASA TM-2002-211646, April 2002.

[5] Martineau, D. G., and Georgala, J. M., "A Mesh Movement Algorithm for High Quality Generalised Meshes," AIAA Paper 2004-0614, Jan. 2004.

[6] Nemec, M., and Zingg, D. W., "Newton-Krylov Algorithm for Aerodynamic Design Using the Navier-Stokes Equations," AIAA Journal, Vol. 40, No. 6, 2002, pp. 1146-1154.

[7] Nemec, M., "Optimal Shape Design of Aerodynamic Configurations: A Newton-Krylov Approach," Ph.D. Thesis, Univ. of Toronto, 2003.

[8] Nemec, M., and Zingg, D. W., "MultiPoint and Multi-Objective Aerodynamic Shape Optimization," AIAA Journal, Vol. 42, No. 6, June 2004, pp. 1057-1065. doi: $10.2514 / 1.10415$

[9] Burgreen, B. W., Baysal, O., and Eleshaky, M. E., "Improving the Efficiency of Aerodynamic Shape Optimization," AIAA Journal, Vol. 32, No. 1, 1994, pp. 69-76.
[10] Burgreen, G. W., and Baysal, O., "Three-Dimensional Aerodynamics Shape Optimization Using Discrete Sensitivity Analysis," AIAA Journal, Vol. 34, No. 9, 1996, pp. 1761-1770.

[11] Jones, W. T., and Samareh-Abolhassani, J. A., "A Grid Generation System for Multidisciplinary Design Optimization,” AIAA Paper 19951689, 1995.

[12] Degand, C., and Farhat, C., "A Three-Dimensional Torsional Spring Analogy Method for Unstructured Dynamic Meshes," Computers and Structures, Vol. 80, Nos. 3-4, Feb. 2002, pp. 305-316. doi:10.1016/S0045-7949(02)00002-0

[13] Liu, X., Qin, N., and Xia, H., "Fast Dynamic Grid Deformation Based on Delaunay Graph Mapping," Journal of Computational Physics, Vol. 211, No. 2, Jan. 2006, pp. 405-423. doi:10.1016/j.jcp.2005.05.025

[14] Stein, K., Tezduyar, T., and Benney, R., "Mesh Moving Techniques for Fluid-Structure Interactions with Large Displacements," Journal of Applied Mechanics, Vol. 70, No. 1, Jan. 2003, pp. 58-63. doi:10.1115/1.1530635

[15] Nielsen, E. J., and Anderson, W. K., "Recent Improvements in Aerodynamic Design Optimization on Unstructured Meshes," AIAA Journal, Vol. 40, No. 6, June 2002, pp. 1155-1163.

[16] Tezduyar, T. E., Behr, M., Mittal, S., and Johnson, A. A., "Computation of Unsteady Incompressible Flows with the Stabilized Finite Element Methods: Space-Time Formulations, Iterative Strategies and Massively Parallel Implementations," New Methods in Transient Analysis, edited by P. Smolinski, W.K. Liu, G. Hulbert, and K. Tamma, Vol. 143, American Society of Mechanical Engineers, New York, 1992.

[17] Squire, W., and Trapp, G., "Using Complex Variables to Estimate Derivatives of Real Functions," SIAM Review, Vol. 40, No. 1, March 1998, pp. 110-112. doi:10.1137/S003614459631241X

[18] Martins, J. R. R. A., Sturdza, P., and Alonso, J. J., "The Complex-Step Derivative Approximation," ACM Transactions on Mathematical Software, Vol. 29, No. 3, Sept. 2003, pp. 245-262. doi: $10.1145 / 838250.838251$

[19] Griewank, A., Evaluating Derivatives: Principles and Techniques of Algorithmic Differentiation, Society for Industrial and Applied Mathematics, Philadelphia, 2000.

[20] Pironneau, O., "On Optimum Design in Fluid Mechanics," Journal of Fluid Mechanics, Vol. 64, 1974, pp. 97-110. doi: $10.1017 / \mathrm{S} 0022112074002023$

[21] Jameson, A., "Aerodynamic Design via Control Theory," Journal of Scientific Computing, Vol. 3, No. 3, 1988, pp. 233-260. doi:10.1007/BF01061285

[22] Anderson, W. K., and Venkatakrishnan, V., "Aerodynamic Design Optimization on Unstructured Grids with a Continuous Adjoint Formulation," Computers and Fluids, Vol. 28, No. 4, 1999, pp. 443 480 .

doi:10.1016/S0045-7930(98)00041-3

[23] Kim, H., Obayashi, S., and Nakahashi, K., "Flap-Deflection Optimization for Transonic Cruise Performance Improvement of Supersonic Transport Wing," Journal of Aircraft, Vol. 38, No. 4, 2001, pp. 709-717. doi:10.1016/S0045-7930(98)00041-3

[24] Kim, H., Sasaki, D., Obayashi, S., and Nakahashi, K., "Aerodynamic Optimization of Supersonic Transport Wing Using Unstructured Adjoint Method," AIAA Journal, Vol. 39, No. 6, 2001, pp. 1011-1020.

[25] Martins, J. R. R. A., Alonso, J. J., and Reuther, J. J., "A CoupledAdjoint Sensitivity Analysis Method for High-Fidelity Aero-Structural Design," Optimization and Engineering, Vol. 6, No. 1, March 2005, pp. 33-62. doi:10.1023/B:OPTE.0000048536.47956.62

[26] Le Moigne, A., and Qin, N., "Variable-Fidelity Aerodynamic Optimization for Turbulent Flows Using a Discrete Adjoint Formulation," AIAA Journal, Vol. 42, No. 7, 2004, pp. 1281-1292. doi:10.2514/1.2109

[27] Bischof, C. H., Mauer, A., Jones, W. T., and Samareh, J., "Experiences with Automatic Differentiation Applied to a Volume Grid Generation Code," Journal of Aircraft, Vol. 35, No. 4, 1998, pp. 569-573.

[28] Maute, K., Nikbay, M., and Farhat, C., "Sensitivity Analysis and Design Optimization of Three-Dimensional Non-Linear Aeroelastic Systems by the Adjoint Method," International Journal for Numerical Methods in Engineering, Vol. 56, No. 6, Feb. 2003, pp. 911-933. doi:10.1002/nme.599

[29] Nielsen, E. J., and Park, M. A., "Using an Adjoint Approach to Eliminate Mesh Sensitivities in Computational Design," AIAA Journal, Vol. 44, No. 5, 2005, pp. 948-953. doi: $10.2514 / 1.16052$ 
[30] Mavriplis, D. J., "Multigrid Solution of the Discrete Adjoint for Optimization Problems on Unstructured Meshes," AIAA Journal, Vol. 44, No. 1, Jan. 2006, pp. 42-50. doi:10.2514/1.15696

[31] Sadrehaghighi, I., Smith, R. E., and Tiwari, S. N., "Grid Sensitivity and Aerodynamic Optimization of Generic Airfoils," Journal of Aircraft, Vol. 32, No. 6, 1995, pp. 1234-1239.

[32] Korivi, V. M., Newman, P. A., and Taylor, A. C., "Aerodynamic Optimization Using Sensitivity Derivatives from a Three-Dimensional Supersonic Euler Code," Journal of Aircraft, Vol. 35, No. 3, 1998, pp. 405-411.

[33] Barger, R. L., Adams, M. S., and Krishnan, R. R., "Automatic Computation of Euler Marching Grids and Subsonic Grids for Wing-
Fuselage Configurations," NASA TM 4573, July 1994.

[34] Pagaldipti, N., and Chattopadhyay, A., "A Discrete Semianalytical Procedure for Aerodynamic Sensitivity Analysis Including Grid Sensitivity," Computers \& Mathematics with Applications, Vol. 32, No. 3, 1996, pp. 61-71.

doi:10.1016/0898-1221(96)00113-7

[35] Gunzburger, M., "Introduction to the Mathematical Aspects of Flow Control and Optimisation," Inverse Design and Optimisation Methods, von Karman Institute of Fluid Dynamics, Brussels, Belgium, April 1997.

Z. Wang 